\title{
Un nuevo prototipo de stent biliar cubierto (flower stent) frente al stent biliar convencional en la prevención de pancreatitis y colecistitis poststent
}

\section{Comparison of Flower Petal Stenting, a new prototype of covered biliary stent, with conventional biliary stenting for prevention of pancreatitis and post-stent cholecystitis}

\author{
Rodrigo Castaño, MD, ${ }^{*}$ Óscar Álvarez, MD, ${ }^{2}$ Jong Kyun Lee, MD, ${ }^{3}$ Jorge Lopera, MD, ${ }^{4}$ Víctor Calvo, MD. ${ }^{5}$
}

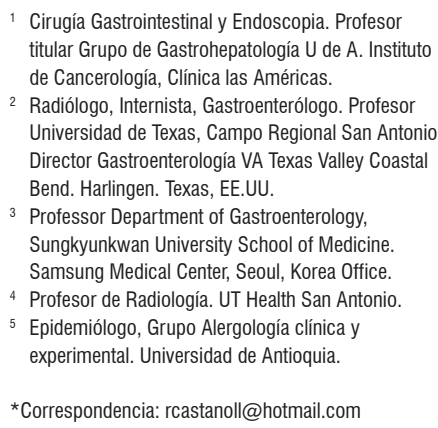

\begin{abstract}
Resumen
Antecedentes y objetivos: la pancreatitis y la colecistitis son complicaciones mayores después de la postura de un stent metálico cubierto en el manejo de la obstrucción biliar distal maligna. Se pretende comparar el uso de un stent biliar cubierto convencional con un nuevo prototipo de stent biliar cubierto de morfología en flor con baja fuerza axial (FA) y fuerza radial (FR), y evaluar el impacto de las cifras de colecistitis y pancreatitis poststent. Métodos: se realizó un estudio prospectivo no aleatorizado en el que se comparó un grupo de 18 pacientes con stent biliar tipo flor (SF) con un grupo de pacientes que recibieron un stent biliar convencional (SC) con una relación 1:2, para el manejo de la obstrucción biliar distal maligna. Se evaluaron datos demográficos; el éxito técnico y clínico; así como la aparición de colecistitis y/o pancreatitis, tasas de migración y/u oclusión. Resultados: se evaluaron 18 pacientes en el grupo con SF (7 hombres, 39 \%) con una edad mediana de 72 años y 36 pacientes ( 16 hombres, $44 \%$ ) en el grupo de SC con una edad mediana de 71 años. Se presentaron 3 casos de colecistitis y 4 casos de pancreatitis, todos ellos en el grupo de SC. Al explorar la asociación de los aspectos clínicos con respecto al tipo de stent, el análisis multivariado mostró diferencias estadísticamente significativas en 4 aspectos: la hemoglobina, la longitud de estenosis, la etiología y la oclusión del stent. Al evaluar los tiempos de supervivencia entre los pacientes con SC y SF, no se hallaron diferencias significativas en las funciones de supervivencia ( $\log$ rank, $p=0,399$ ). En el modelo de riesgos proporcionales de Cox, solo un aspecto mostró diferencias significativas frente a los tiempos de supervivencia: la presencia de metástasis. Conclusiones: el uso de un nuevo prototipo de stent SF (con surcos laterales) con menor FA y FR se relaciona con un menor índice de complicaciones como colecistitis y pancreatitis en comparación con el SC, en la paliación de las estenosis biliares malignas distales sin detrimento de su permeabilidad o migración y sin afectar la supervivencia de estos pacientes.
\end{abstract}

Palabras clave

Stent biliar metálico, obstrucción biliar, colecistitis, pancreatitis.

\section{INTRODUCCIÓN}

Los stents biliares metálicos (SBM) implementados en la colangiografía endoscópica retrógrada (CPRE) han mostrado una mayor permeabilidad que los stents plásticos en el manejo de la obstrucción biliar maligna (OBM) (1-3) e incluso se sugiere que son más costo-efectivos (4), pero algunas complicaciones diferentes a la oclusión del stent pueden estar asociadas con el SBM y pueden afectar la calidad de vida de los pacientes que de base cursan con un pobre pronóstico. Aunque los SBM tienen una mayor permeabilidad, los stents cubiertos previenen el crecimiento tumoral al interior del stent, aunque pueden cursar con una mayor tasa de migración (5). Además, los stents biliares cubiertos pueden ocluir el orificio de drenaje del conducto cístico o del pancreático e inducir colecistitis (6-8) o pancreatitis (9); pero hay datos contrarios, entre ellos 2 recientes metaanálisis que compararon stents cubiertos frente a descubiertos $(9,10)$ 


\begin{abstract}
Background and objectives: Pancreatitis and cholecystitis are major complications following stenting with covered metallic stents to manage malignant distal biliary obstructions. We compare the use of a conventional covered biliary stent with a new prototype of a covered biliary stent with a flower-pedal structure that uses with low axial force and radial force in order to evaluate impacts on cholecystitis and pancreatitis after stenting. Methods: This is a non-randomized prospective comparison of a group of 18 patients stented with a flowerlike biliary stent (FS group) with a group of 36 patients who received conventional biliary stents (CS group) ratio for management of malignant distal biliary obstructions. Demographic data and data about technical and clinical success including the appearance of cholecystitis and/or pancreatitis, migration rates and/or occlusion were evaluated. Results: The 18 FS group patients included 7 men (39\%) and had a median age of 72 years. The 36 CS group patients included 16 men (44\%) and had a median age of 71 years. There were three cases of cholecystitis and four cases of pancreatitis, all of which were in the CS group. Multivariate analysis showed statistically significant differences between the two groups in four clinical areas: hemoglobin, length of stenosis, etiology and occlusion of stents. No significant differences were found in survival time functions (Log Rank, $p=0.399$ ). In Cox's proportional hazards model, the only significant difference with respect to survival times was found on the issue of whether or not metastasis was present. Conclusions: The use of the new prototype flower-like biliary stent with lateral grooves and lower axial force and radial force is related to a lower rate of complications such as cholecystitis and pancreatitis than the use of conventional stents for palliation of distal malignant biliary stenoses. The new stent has no detriments in terms of permeability or migration and did not affect the survival of these patients.
\end{abstract}

\title{
Keywords
}

Metallic biliary stent, biliary obstruction, cholecystitis, pancreatitis.

relacionaron la colecistitis más con la invasión tumoral del cístico o con la misma fuerza expansiva axial y radial del stent que con el uso del stent cubierto (11).

Los SBM se han agrupado de forma simple entre los cubiertos, parcialmente cubiertos o descubiertos. Diversos tipos de SBM con diferentes propiedades mecánicas han llevado a distintos resultados clínicos aun dentro de los grupos de stents cubiertos o descubiertos. 2 propiedades mecánicas son la fuerza axial (FA) y la fuerza radial (FR) de los stents. La FA es la fuerza de recuperación que lleva al stent a enderezarse luego de haberse liberado y se relaciona con la conformabilidad del conducto biliar, y la FR es la fuerza de expansión que garantiza la permeabilidad del stent contra la estenosis $(12,13)$. Un SBM con una alta FA tiende a estar recto dentro del conducto biliar, comprime el conducto biliar y lo puede llevar a angulaciones (14). Previos estudios han demostrado que los SBM con alta tasa de FA se asocian con una alta tasa de pancreatitis, probablemente asociados con la compresión del orificio pancreático por el stent (15).

Diferentes estudios han mostrado que el compromiso tumoral del conducto cístico o el uso del stent cubierto pueden inducir colecistitis, pero no han mostrado la manera de prevenirla $(7,8,16)$. La incidencia de colecistitis con el uso de los SBM cubiertos es de 4,2 \% a 11,5 \% en el manejo de la OBM distal $(11,17)$. Aunque no se ha descrito sepsis de origen pancreático, la pancreatitis se ha reportado hasta el en 9,3\% de casos de manejo con stents cubiertos de la OBM. Estas complicaciones en los pacientes con obs- trucciones malignas manejadas con SBM cubierto pueden inducir la necesidad de una nueva intervención endoscópica o quirúrgica, lo que además de afectar la calidad de vida del paciente puede dilatar o descontinuar tratamientos como la quimioterapia o radioterapia.

La colecistitis después de la postura de un stent biliar puede ocurrir por colonización bacteriana de la bilis luego de la manipulación endoscópica o del mal vaciamiento de la vesícula causada por la obstrucción del cístico por la invasión tumoral, lo que permite el llenado de la vesícula con medio de contraste no estéril. La colecistitis que se desarrolla a los días de la colocación de un stent puede asociarse con la oclusión del conducto cístico por la fuerza expansiva del stent metálico cubierto, situación en la cual tiene una alta injerencia la FA del stent. Tres estudios $(7,8,16)$ han mostrado la asociación del compromiso tumoral del cístico con la aparición de colecistitis, pero no se han reportado las medidas necesarias para prevenirla.

Se ha documentado en un estudio en cerdos con un stent con una morfología diferente, tipo flor (SF), que el flujo de la bilis es mayor, los cambios inflamatorios histológicos son menores y la proteína C-reactiva (PCR) es menor con el uso del nuevo stent (18).

No existe el stent ideal para su uso en la vía biliar y el objetivo después de su aplicación es logar la máxima permeabilidad en el tiempo sin complicaciones inherentes a su uso. Desde el punto de vista mecánico, las propiedades de un stent biliar ideal es proveer una baja FA con una alta FR y con sistemas antimigración. Muchas complicaciones 
asociadas con los stents pueden resolverse en el futuro con la implementación del stent ideal.

Con la intención de prevenir las complicaciones inherentes al uso de SBM cubiertos como la colecistitis y la pancreatitis se realizó un estudio multicéntrico de seguimiento prospectivo en el que se evaluó la factibilidad técnica y la seguridad con el uso de un nuevo stent con la morfología de flor, stent en flor (SF) con una baja FA y FR, y se comparó con un grupo manejado con un SBM cubierto convencional, stent convencional (SC) con mayor FA y FR, y morfología circular.

\section{MATERIALES Y MÉTODOS}

\section{Fase experimental}

Los stents metálicos cubiertos tienen el riesgo de obstruir el conducto cístico o el ducto principal pancreático, debido a su alta FR y su cobertura, lo que en la clínica se traduce en una mayor posibilidad de colecistitis y pancreatitis. Un stent metálico autoexpandible cubierto y en forma de flor semeja una flor de 5 pétalos con surcos laterales, se ha diseñado para evitar la oclusión del conducto cístico y su aplicación inicialmente se evaluó en modelos porcinos de alrededor de 30 kilos.
En Corea, el Dr. Young Sik Woo (18) le aplicó en forma aleatoria el SF o el SC a 14 cerdos (7 por grupo). Los stents se ubicaron de forma que ocluyeran el drenaje a través del cístico, obstaculizando el drenaje de la vesícula. El drenaje de la vesícula se midió a las 24, 48, 120 y 168 horas después de la implantación. Se tomaron exámenes de sangre al segundo y séptimo día luego de la implantación de los stents. Los animales sobrevivieron a los 7 días fijados como plazo para el sacrificio y la correspondiente evaluación histológica (Figura 1).

Luego de la administración de $25 \mathrm{~mL}$ de contraste en la vesícula biliar, los stents se ubicaron en la posición requerida en todos los casos y no se presentaron complicaciones con la intervención. A las 48 horas, la tasa de drenaje de la vesícula fue mayor en los casos con el SF que en el grupo con el SC sin una diferencia significativa (71,4 frente a 28,6 $\%, p=0,28$ ). Se presentó una migración temprana (dentro los 7 días) del stent en cada grupo. Los SC se relacionaron con unas cifras más altas de PCR como marcador de inflamación $(35,2$ frente a 20,5 $\mu \mathrm{g} / \mathrm{dL}, \mathrm{p}=0,03)$.

En el momento de la laparotomía se removieron en bloque el hígado, vesícula biliar y conductos biliares, y duodeno. La vesícula se abrió longitudinalmente y se tomaron muestras para el estudio histológico. No se evidenciaron signos de infección o peritonitis en el tejido hepático, ni duodenal,
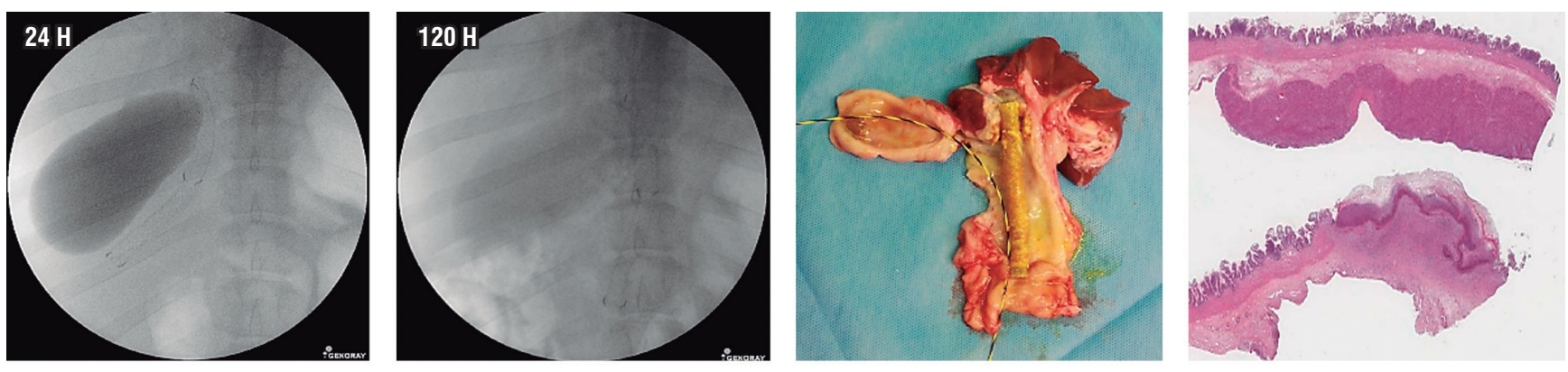

SC y secuencia en el vaciamiento del contraste, aspectos quirúrgicos e histológicos
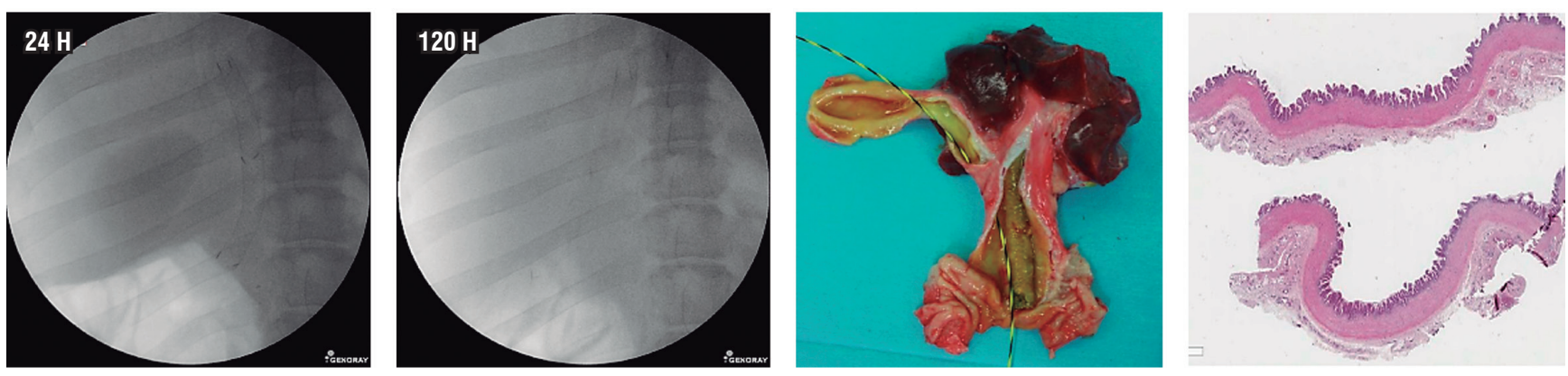

SF y secuencia en el vaciamiento del contraste, aspectos quirúrgicos e histológicos

Figura 1. Aspectos a la CPRE, cirugía e histología del SC y el SF. 
colédoco o la vesícula al momento de la laparotomía. El estudio histológico se revisó de forma ciega utilizando una escala de puntuación que evalúa la inflamación ( 1 = mínima, $2=$ confinada a la mucosa, 3 = compromiso hasta el músculo), severidad del infiltrado neutrófilo $(0=$ ausente, $1=$ leve, $2=$ moderado y $3=$ severo) y la presencia de úlceras en la mucosa $(0=$ ausentes, $1=$ focales y $2=$ difusas $)$.

3 cerdos en el grupo del SC presentaron al momento del sacrificio una vesícula distendida con material bilioso maloliente y con cambios histológicos inflamatorios de leves a moderados, con una inflamación y una puntuación global (con infiltrado neutrófilo y ulceraciones en mucosa) significativamente mayores en el SC ( $p=0,04$ y $p=0,03)$.

Con base en estos resultados experimentales preliminares se implementó el estudio clínico que se detalla a continuación.

\section{Población de estudio}

Los pacientes que se presentaron con una OBM entre marzo de 2015 y febrero de 2016 se recogieron en una base de datos prospectiva, cuyos criterios de inclusión fueron: presencia de ictericia obstructiva, OBM demostrada por tomografía axial computarizada (TAC) y/o resonancia magnética de abdomen, apariencia radiológica de OBM a la CPRE, no ser candidato para una resección quirúrgica por el estado tumoral o los riesgos quirúrgicos, ser mayor de 18 años de edad, vesícula in situ y firma del consentimiento informado diseñado para este proyecto. Los criterios de exclusión fueron: alguna contraindicación para realizar la CPRE; presencia de un SBM, cirugía o algún procedimiento de drenaje percutáneo; presencia de hepatitis u otra enfermedad hepática que curse con ictericia; y paciente colecistectomizado o con sospecha de cáncer de vesícula.

Los siguientes datos clínicos se incluyeron en la base de datos: aspectos demográficos, comorbilidades, estado del tumor, antecedente de quimioterapia y/o radioterapia, detalles de la CPRE, tipo y número de stents utilizados y eventos adversos de acuerdo con lo recomendado por las guías de la Asociación Americana de Endoscopia (ASGE) (19).

\section{Tipos de stents utilizados}

Se comparó el nuevo SF en sus extremos con surcos laterales, de $10 \mathrm{~mm}$ de diámetro por $80 \mathrm{~cm}$ de longitud, cubiertos por politetrafluoroetileno con una baja FA y FR; con un SC de Boston Scientific con la misma cobertura y los mismos diámetros, con una alta FA y FR, montados ambos en un mecanismo introductor de $8 \mathrm{Fr}$. El SF está hecho de nitinol y presenta en los extremos la forma de una flor de 5 pétalos con surcos paralelos en su eje largo, diseñado específicamente para prevenir la oclusión del conducto cístico o pancreático y facilitar el drenaje de la vesícula biliar y el conducto pancreático (Figura 2).

Además, el SF presentaba una alta conformabilidad, definida como la capacidad del stent de adaptarse completamente a la anatomía de la región donde es liberado, independiente de la irregularidad o tortuosidad de la zona por la presencia de la tumoración. Esto significa que una vez el stent en flor se deforma en su eje axial, la prótesis no regresa inmediatamente a su forma inicial.

\section{FA y FR de los stents utilizados}

Los stents metálicos utilizados en el presente estudio se clasifican de acuerdo con su FA (definida como la fuerza de recuperación necesaria para mantenerse el stent en forma derecha después de haber sido doblado) y su FR, en la que se evalúan 2 aspectos: la fuerza necesaria para contraer el stent y, la más relevante clínicamente, la fuerza de expansión del stent una vez este ha sido colapsado. Se realizaron mediciones de la FA con el SF y el SC, y se demostró una diferencia hasta 4 veces mayor de la FA del SC sobre el SF (Figura 3).
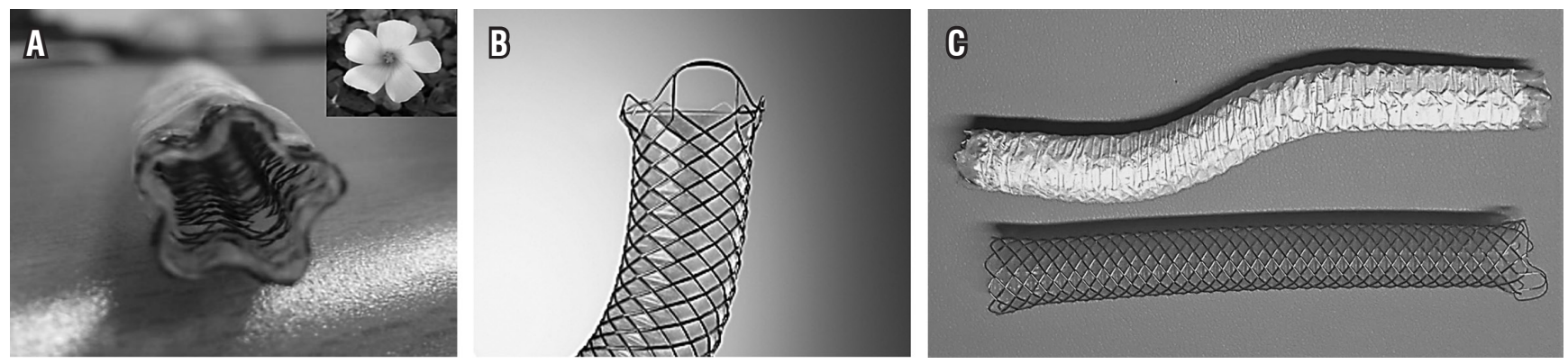

Figura 2. Tipos de stents cubiertos utilizados en el estudio. A. SF cubierto de forma pentagonal con surcos orientados en paralelo a lo largo de su eje mayor. B. Stent metálico estándar de Boston Scientific, Wallstent, de forma cilíndrica y totalmente cubierto. C. El SF (en la parte superior) presenta una mayor conformabilidad (conserva la forma en S) con una menor FR y FA con respecto al SC. 


\section{Diseño del estudio}

Este es un ensayo abierto (open label) pareado por edad (1 SF por 2 SC) realizado en 2 instituciones de la ciudad de Medellín. Una vez el paciente cumpliera los criterios de inclusión y se descartaran los criterios de exclusión, se obtenía el consentimiento informado; al paciente se le ponía el SF y los datos del grupo control se obtenían de una base de datos prospectiva de las instituciones.

Los hallazgos en los pacientes con estenosis biliares malignas que recibieron el SF se compararon con un control pareado por edad ( \pm 2 años) y el diagnóstico de estenosis biliar maligna que recibiera una CPRE con la colocación de un SC.

\section{Técnica endoscópica}

Todos los procedimientos se realizaron usando protocolos estandarizados por endoscopistas con largo recorrido en la endoscopia biliar (>10 años). A los pacientes se les aplicó antibióticos profilácticos de amplio espectro. Se utilizó una técnica estándar para canular la vía biliar y se inyectó el contraste para identificar la posición y longitud de la estenosis. No se realizó una esfinterotomía biliar rutinaria en ningún paciente. Con la ayuda de la fluoroscopia se determinaron la longitud de la estenosis, ubicación del tumor, compromiso tumoral o no del conducto cístico y grado de llenado con contraste de la vesícula biliar.

Se puso el stent con el nuevo diseño liberándolo por tracción del mecanismo introductor. La longitud del stent se determinó de acuerdo con la longitud de la estenosis, posicionando el centro del stent en el centro de la estenosis y ubicando el extremo duodenal del stent 10 a $15 \mathrm{~mm}$ por fuera de la ampolla.
No se requirió del uso del stent pancreático profiláctico en ningún paciente, ni se administraron medicamentos profilácticos para la aparición de pancreatitis aguda.

\section{Definición de eventos y seguimiento}

Para confirmar si el drenaje biliar es exitoso se tenían pruebas de la función hepática de la última semana antes de poner el stent, y estas pruebas se repetían al segundo, quinto y séptimo día luego de su colocación, con seguimiento a los 30 días y luego mensual. Los pacientes se revisaban al mes personalmente o por contacto telefónico, con pruebas de función hepática. Los pacientes que se perdieron del seguimiento se analizaron con base en la intención de tratar y se censuraron al momento de su último contacto para seguimiento.

El éxito técnico se definió como la inserción y ubicación exitosa del stent, basado en la confirmación radiológica durante la CPRE. El éxito clínico se definió como la caída de más del $50 \%$ de la bilirrubina en los primeros 7 días después de la colocación del stent. Todos los efectos adversos relacionados con el procedimiento se registraron. Los efectos adversos tempranos se definieron como los que suceden en los primeros 30 días luego de la liberación del stent, y los tardíos los que suceden después de este período. Se consideró una disfunción del stent en el paciente con ictericia obstructiva y dilatación de la vía biliar por imágenes (ecografía, TAC o resonancia) con o sin colangitis. La disfunción del stent motivó una nueva CPRE para valorar y corregir la causa de la disfunción. Si la ictericia recurrente cursa con fiebre, se realiza un CPRE urgente, aunque las imágenes no mostraran una dilatación biliar. La oclusión del stent puede darse por crecimiento tumoral encima del mismo o por crecimiento a través del stent. La migración proximal o distal del stent se
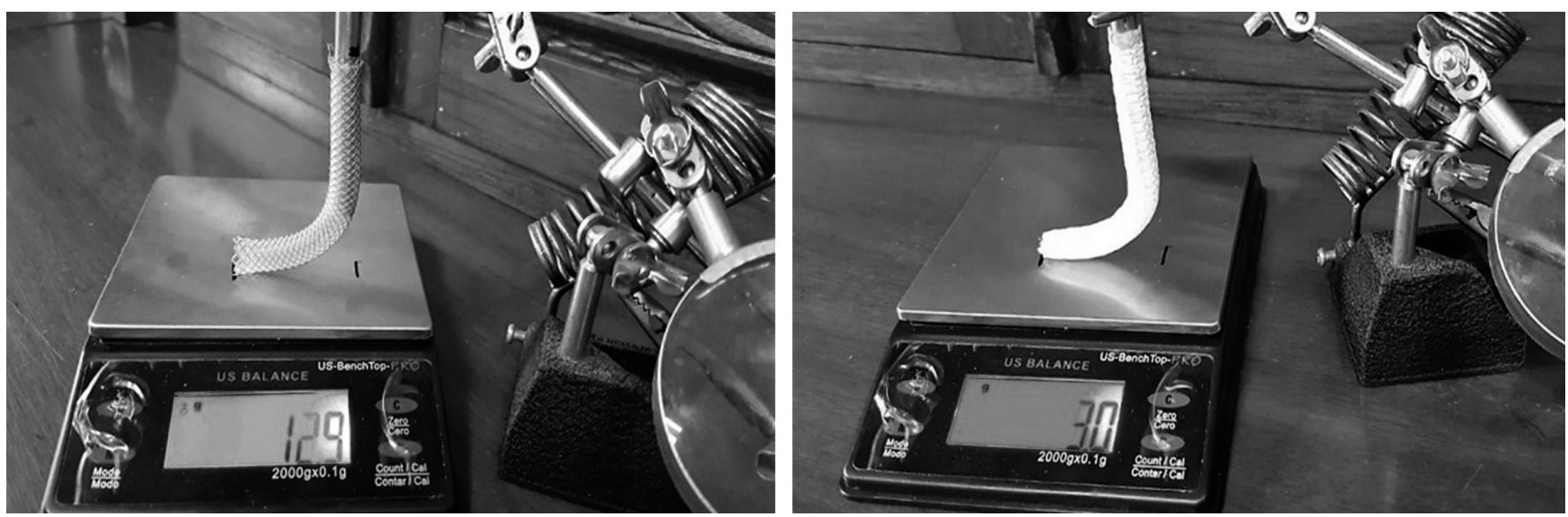

Figura 3. Medición de la fuerza axial de los 2 stents, siendo tres veces mayor en el stent convencional sobre el stent flower $(12,9$ vs. 3,0). 
define como el desplazamiento del mismo por encima o por debajo de la OBM después de su liberación.

\section{Medidas de desenlace}

Los resultados primarios a evaluar son la aparición de colecistitis y/o pancreatitis. Después de la liberación del stent, definida como la presencia de dolor abdominal superior acompañado de sensibilidad en cuadrante superior derecho (signo de Murphy) por clínica o por ecografía, con hallazgo de engrosamiento de la pared de la vesícula superior a $3 \mathrm{~mm}$ con o sin colección perivesicular.

La pancreatitis se definió con base en criterios estándar (20), como un dolor abdominal nuevo o que empeoraba después de la colocación del stent con aumento de los niveles de amilasa o lipasa 3 veces por encima de los valores normales, con o sin evidencia radiográfica de pancreatitis. La severidad se determinó de la siguiente manera: leve, si la estancia hospitalaria se prolongaba no más de 3 días de lo planeado; moderada, de 4 a 10 días de estancia hospitalaria; o severa, cuando la estancia era mayor de 10 días, iba a cuidado intensivo o a cirugía.

Análisis secundarios son el éxito técnico y clínico (documentado por resultados de laboratorio), causas de disfunción del stent que ameriten reintervención, efectos adversos y supervivencia. La supervivencia se midió desde el momento de la postura del stent hasta la fecha de su muerte o la pérdida del seguimiento.

\section{Análisis estadístico}

Para el análisis descriptivo de los aspectos sociodemográficos y clínicos se utilizaron distribuciones absolutas, relativas e indicadores de resumen como los cuartiles y el rango intercuartílico. Se estableció el criterio de normalidad de las variables continuas por medio de la prueba de ShapiroWilk y, con base en esta, se utilizó la prueba U-Mann Whitney para la diferencia entre los 2 tipos de stents.

Para establecer la relación entre los aspectos clínicos y el tipo de stent se aplicó la prueba de chi cuadrado $\left(\chi^{2}\right)$ de independencia de Pearson o la prueba exacta de Fisher; de igual manera, se evaluó la fuerza de asociación por medio del riesgo relativo (RR) con sus respectivos intervalos de confianza del 95 \% (IC $95 \%$ ). Se aplicó regresión multivariada como método exploratorio para evaluar la influencia de aspectos sociodemográficos y clínicos según el tipo de stent a través de un modelo lineal generalizado (GML) de regresión de Poisson con varianza robusta para ajustar el RR y sus IC $95 \%$ por múltiples variables.

Para evaluar la supervivencia de los pacientes de acuerdo con el tiempo de permeabilidad del stent y la supervivencia global, se utilizó el método de Kaplan-Meier, el cual propor- cionó la probabilidad de morir de un paciente en un tiempo determinado a partir del momento de la colocación del stent. El evento de interés para este análisis fue la muerte (condición de no censura). Para determinar la diferencia o no de los tiempos de supervivencia con respecto a algunos aspectos sociodemográficos y clínicos, se aplicó la prueba del rango semilogarítmico (log rank test) con sus estimativos Hazard ratio (HR) y los respectivos IC $95 \%$, y ajustados por medio de un modelo multivariado exploratorio de riesgos proporcionales de Cox. Los valores de $p<0,05$ se consideraron estadísticamente significativos. Todos los análisis estadísticos se realizaron con el software SPSS (versión 21).

\section{Aspectos éticos}

El stent utilizado es un prototipo con los mismos materiales que los SC, constituidos por nitinol y cubiertos por politetrafluoroetileno. El protocolo y uso del stent fue aprobado por el comité de investigación de la entidad hospitalaria que comercializa el stent.

\section{RESULTADOS}

Los stents se liberaron exitosamente en los 54 pacientes y cruzaban el orificio de implantación del conducto cístico, sin evidencia de complicaciones mayores inmediatas. Las características de base de ambos grupos en estudio fueron muy similares. En los siguientes 30 días luego de la liberación del stent se realizaron controles de laboratorio al día 7 y al día 30, continuando con controles de laboratorio mensuales. No se presentaron pérdidas del seguimiento en ninguno de los grupos. Las causas de la OBM fueron cáncer de páncreas $(\mathrm{n}=37)$, colangiocarcinoma $(\mathrm{n}=14)$ y tumor periampular $(n=2)$. En total, 28 pacientes recibieron terapia adyuvante luego de la inserción del stent: 10 (55,6\%) en el grupo de SF y 18 en el grupo de SC (50 \%) (Tabla 1).

Cuando se evaluó la asociación de estos factores sociodemográficos y clínicos con respecto al tipo de stent, se observaron diferencias estadísticamente significativas en 4 aspectos: la hemoglobina (más baja en SF), la longitud de estenosis (más larga en SF), la etiología (más colangiocarcinoma en SC) y la oclusión del stent (menos en SF) (Tabla 1).

\section{Colecistitis aguda}

La colecistitis aguda luego de la colocación del stent ocurrió en 3 pacientes a los 6, 8 y 16 días (media 10 días), todas ellas en el grupo con el stent estándar. La colecistitis se mejoró con manejo conservador con antibióticos parenterales en un paciente, y 2 de ellos requirieron la colecistostomía con apoyo radiológico. No se realizó colecistectomía en ninguno de los pacientes. Característicamente, los 
Tabla 1. Características de base de los grupos de pacientes con SF y SC

\begin{tabular}{|c|c|c|c|c|c|}
\hline & \multicolumn{2}{|c|}{ Tipos de stent } & \multirow[t]{2}{*}{ Valor de $p^{*}$} & \multirow[t]{2}{*}{$\mathrm{RR}^{*}$} & \multirow[t]{2}{*}{ IC $95 \%{ }^{*}$} \\
\hline & $S C(n=36)$ & $S F(n=18)$ & & & \\
\hline Edad $^{* *}$ & $71(7)$ & $72(3)$ & 0,370 & 1,01 & 0,98 a 1,04 \\
\hline Sexo (hombre/mujer) & $16 / 20$ & $7 / 11$ & 0,531 & 1,18 & 0,69 a 2,00 \\
\hline Hemoglobina** & $10,4(1)$ & $9,9(2)$ & 0,004 & 1,36 & 1,10 a 1,67 \\
\hline Leucocitos ${ }^{\star *}$ & $6531(1855)$ & $6425(1375)$ & 0,627 & 1 & 0,99 a 1,01 \\
\hline Bilirrubina total $\mathrm{mg} / \mathrm{dL}^{* *}$ & $4,4(1,4)$ & $4,3(1,2)$ & 0,336 & 0,90 & 0,73 a 1,11 \\
\hline Fosfatasa alcalina** & $258(66)$ & $224(102)$ & 0,922 & 0,99 & 0,99 a 1,01 \\
\hline Albúmina** & $2,7(0,6)$ & $2,5(0,6)$ & 0,801 & 1,10 & 0,52 a 2,32 \\
\hline Stent plástico previo & 5 & 2 & 0,663 & 1,15 & 0,62 a 2,12 \\
\hline Pancreatograma & 7 & 2 & 0,222 & 1,62 & 0,74 a 3,55 \\
\hline Compromiso del cístico & 7 & 2 & 0,062 & 2,97 & 0,95 a 9,29 \\
\hline Colelitiasis & 4 & 4 & 0,510 & 0,77 & 0,35 a 1,68 \\
\hline Longitud de estenosis $(\mathrm{cm})^{\star *}$ & $3(1)$ & $4(2)$ & 0,001 & 0,63 & 0,47 a 0,84 \\
\hline \multicolumn{6}{|l|}{ Etiología: } \\
\hline Cáncer de páncreas & 25 & 12 & 0,036 & 4,84 & 1,11 a 21,08 \\
\hline Colangiocarcinoma & 10 & 4 & 0,010 & 8,31 & 1,67 a 41,18 \\
\hline Cáncer periampular & 1 & 2 & & & \\
\hline Colecistitis aguda & 3 & 0 & 0,699 & 1,21 & 0,45 a 3,22 \\
\hline Pancreatitis aguda leve & 4 & 0 & 0,738 & 1,15 & 0,51 a 2,55 \\
\hline Migración & 2 & 1 & 0,543 & 1,34 & 0,52 a 3,48 \\
\hline Oclusión & 5 & 2 & 0,048 & 1,56 & 1,01 a 2,42 \\
\hline Metástasis & 16 & 7 & 0,470 & 1,34 & 0,61 a 2,94 \\
\hline Quimioterapia & 17 & 11 & 0,055 & 0,49 & 0,24 a 1,01 \\
\hline
\end{tabular}

*Ajustados por el modelo de regresión de Poisson (GLM). ${ }^{* *}$ Los valores se presentan en mediana (rango intercuartílico).

3 pacientes que presentaron colecistitis aguda presentaban compromiso tumoral a la altura de la implantación del cístico. La inyección del contraste en la vesícula no se relacionó con la aparición de colecistitis. La presencia de cálculos en la vesícula no determinó una mayor probabilidad de colecistitis en estos pacientes.

\section{Pancreatitis aguda}

Se presentó en 4 de los 36 pacientes $(11,1 \%)$ con SC y el inicio se dio en las primeras 24 horas después de la colocación del stent. Todos los casos de pancreatitis fueron leves de acuerdo con los criterios del consenso citado. El manejo conservador sin remoción del stent llevó a la mejoría de todos los pacientes. El promedio de ayuno de estos pacientes fue de 5 días ( 2 a 8 días). 2 de los pacientes con pancreatitis tenían antecedente de canulación y/o inyección del contraste en el conducto pancreático, y en 3 de los 4 pacientes el compromiso tumoral era extrapancreático (colangiocarcinoma).

\section{Laboratorio}

Se compararon los resultados del análisis previo a la colocación del stent de la hemoglobina, los leucocitos, la bilirrubina total, la fosfatasa alcalina y la albúmina; y se encontraron diferencias significativas solo en el nivel de hemoglobina, los pacientes con SF tenían 0,5 g/dL menos de hemoglobina que los de SC.

\section{Otras complicaciones}

La migración de los stents fue similar para ambos grupos; sin embargo, la posibilidad de oclusión fue mayor para el grupo con el SC, diferencia que fue estadísticamente significativa ( $\mathrm{p}=0,048$; RR: 1,56; IC $95 \%$ : 1,01 a 2,42).

\section{Análisis de supervivencia}

Para el análisis se consideró el tiempo que transcurre desde la fecha de colocación del stent hasta la fecha de muerte. El 
evento de interés para este análisis fue la muerte (condición de no censura) por causa básica relacionada con patología biliar maligna.

Cuando se evaluaron los tiempos de supervivencia entre los pacientes con SC y SF no se hallaron diferencias significativas en las funciones de supervivencia ( $\log r a n k$, $p=0,399)$. El tiempo de supervivencia en el $50 \%$ de los pacientes con SF fue de 395 días con una probabilidad de muerte de 0,471; y el tiempo de supervivencia en el $50 \%$ de los pacientes con SC fue de 351 días con una probabilidad de muerte de 0,460 (Figura 4).

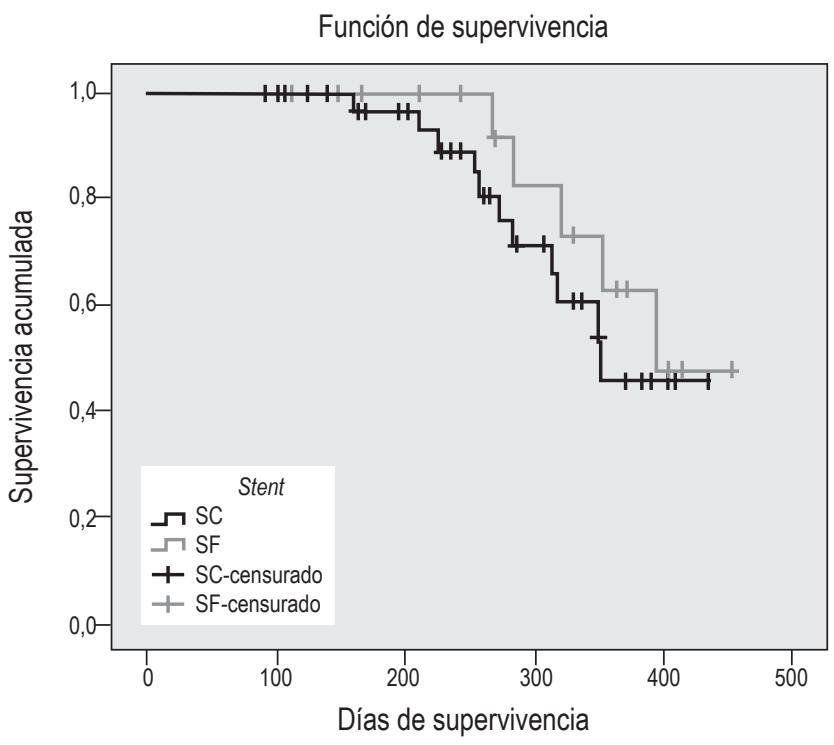

Figura 4. Función de supervivencia de los pacientes con patología biliar maligna según el tipo de stent.

\section{Análisis de riesgos proporcionales de Cox}

De acuerdo con la prueba de rango semilogarítmico (log rank test), por criterios de plausibilidad, de conocimiento y de experiencia de los investigadores se ingresaron las siguientes variables al modelo: tipo de stent, grupo de edad, sexo, stent plástico previo, colelitiasis, CA páncreas, migración, metástasis y quimioterapia. El modelo es adecuado de acuerdo con el método de máxima verosimilitud ( $\mathrm{p}=$ 0,017 ) y se presenta tanto la evaluación global del supuesto de riesgos proporcionales como la evaluación de cada covariable dentro del modelo utilizando los residuos de Schoenfeld (Tabla 2).

De acuerdo con el modelo final, solo un aspecto mostró diferencias significativas frente a los tiempos de supervivencia: la presencia de metástasis; por tanto, el riesgo de morir en el grupo de pacientes con metástasis es 41,7 veces el riesgo de muerte en relación con los pacientes sin metástasis en todo el tiempo y ajustado por las demás variables (Tabla 2).
La evaluación global de los supuestos de riesgos proporcionales $(\mathrm{p}=0,1722)$ y de cada variable del modelo ( $\mathrm{p}$ $>0,05$ ) cumple con el supuesto de acuerdo con los residuos de Schoenfeld.

\section{DISCUSIÓN}

En general, el drenaje endoscópico con SBM cubiertos es aceptado como la primera línea de terapia paliativa en pacientes con OBM distal e irresecable, por ser poco invasivo y por su más larga permeabilidad, que es superior a los stents plásticos. Sin embargo, los SBM cubiertos se asocian con diversas complicaciones que afectan la calidad de vida de estos pacientes ya comprometidos por su tumor de base. La pancreatitis y colecistitis agudas son 2 complicaciones asociadas con el manejo con SBM de la estenosis biliar, las tasas de pancreatitis asociadas con el SBM oscilan entre $1 \%$ y $8 \%$ y las de colecistitis entre $6 \%$ y $12 \%(8,15,20)$. En nuestro estudio estas tasas se ajustan a lo reportado en la literatura.

La prevención de estas complicaciones es tan importante como la permeabilidad del stent a largo plazo. Este estudio multicéntrico y con una base de datos de seguimiento prospectivo revela que el compromiso tumoral del cístico y el uso de stent con alta FA (otros estudios con stents más cortos) se relacionan con un mayor riesgo de colecistitis y pancreatitis.

2 estudios retrospectivos $(8,16)$ sugieren que la asociación del compromiso tumoral del cístico y el conducto de Wirsung con la colecistitis y pancreatitis es por la estenosis del orificio o la obstrucción local con pérdida de la elasticidad debido a la presencia del compromiso tumoral y la compresión por el stent cubierto que desplaza el tumor con dificultad para el paso del flujo biliar desde la vesícula. Por el contrario, en los casos con un cístico sin compromiso tumoral, la elasticidad del mismo permite el espacio necesario para el paso de la bilis desde la vesícula. Teóricamente, un stent descubierto está menos propenso a presentar colecistitis. Sin embargo, la incidencia de colecistitis o pancreatitis no difiere significativamente al comparar stents cubiertos frente a los descubiertos. Por tanto, la FA, más que la presencia del cubrimiento del stent, puede producir la compresión del cístico con compromiso tumoral e impedir el paso de bilis o jugo pancreático con el riesgo de colecistitis o pancreatitis (11).

Se ha considerado que las propiedades mecánicas de los stents pueden afectar el pronóstico clínico, y las 2 propiedades mecánicas más relevantes en los stents son la FR y la FA (12). Los estudios muestran que los stents con alta FA se asocian con colecistitis y pancreatitis (15). Una alta FR no se ha relacionado con colecistitis mientras la FA sí, esto se explica porque los stents con alta FA pueden causar desviación del orificio del conducto cístico con mayor compresión del orificio; contrario a la expansión radial, que es uniforme a lo largo del eje de la vía biliar (11) (Figura 5). 
Tabla 2. Análisis de riesgos proporcionales de Cox a pacientes con patología biliar maligna

\begin{tabular}{lcccc}
\hline & Valor de $p$ crudo & HR (IC 95 \%) crudo & Valor de $p$ ajustado & HR (IC 95 \%) ajustado \\
\hline Tipo de stent (SC) & 0,399 & $1,58(0,54$ a 4,59) & 0,579 & $1,46(0,38$ a 5,52) \\
Grupo de edad (>70 años) & 0,045 & $0,34(0,12$ a 0,97$)$ & 0,140 & $0,36(0,09$ a 1,39) \\
Sexo (hombre) & 0,354 & $0,61(0,22$ a 1,72) & 0,814 & $0,85(0,23$ a 3,15) \\
Stent plástico previo & 0,907 & $1,07(0,30$ a 3,79) & 0,196 & $3,21(0,55$ a 18,87) \\
Colelitiasis & 0,339 & $1,85(0,52$ a 6,60) & 0,180 & $2,98(0,60$ a 14,78) \\
CA páncreas & 0,528 & $1,44(0,46$ a 4,47) & 0,646 & $1,40(0,33$ a 5,96) \\
Migración & 0,947 & $1,07(0,14$ a 8,21) & 0,284 & $5,11(0,26$ a 101,2) \\
Metástasis & 0,003 & $6,72(1,88$ a 23,89) & 0,005 & $41,7(3$ a 579,4$)$ \\
Quimioterapia & 0,081 & $2,74(0,88$ a 8,54) & 0,207 & $0,25(0,03$ a 2,16) \\
\hline
\end{tabular}

Shimizu (16) investigó la asociación de la FA y FR con la aparición de pancreatitis y/o colecistitis sin encontrar una relación directa. Es posible que la incidencia de colecistitis $(n=4)$ o pancreatitis $(n=8)$ en su población de estudio ( $n$ $=72$ ) haya sido demasiado pequeña para evaluar las diferencias de las características de los stents cubiertos, dado el hecho de que los 4 pacientes que sufrían de colecistitis sí recibieron stents cubiertos con alta FA.

Aunque la longitud del stent se selecciona de acuerdo con la extensión de la estenosis, en el presente estudio se utilizaron stents no mayores a $10 \mathrm{~cm}$ de longitud. Se ha reportado la ventaja de utilizar stents más largos para reducir las complicaciones relacionadas con el mismo, previniendo las colecistitis y la oclusión por sobrecrecimiento tumoral. Los stents más cortos se han relacionado con una mayor tasa de colecistitis $(12,21)$. Si se coloca un stent corto, la FA será mayor porque la distancia entre la estenosis y el extremo del stent es menor. Se ha informado la ventaja de usar stents largos para disminuir las complicaciones (21). Un stent más largo puede prevenir

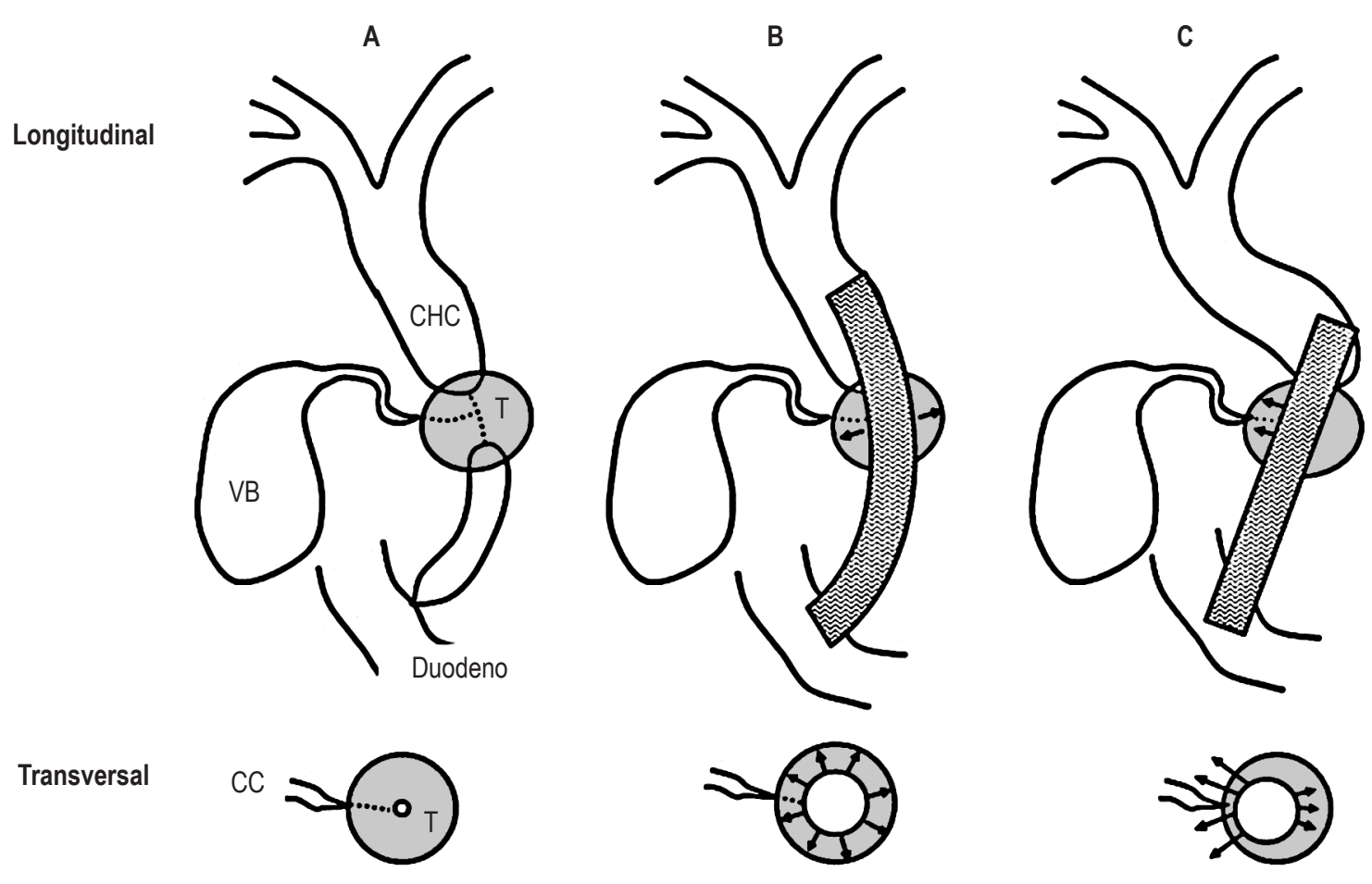

Figura 5. Vista longitudinal y transversal de los efectos de la FA del stent metálico liberado en pacientes con compromiso tumoral del conducto cístico (11). A. Antes de la colocación del stent. B. Después de colocar un stent con baja FA. C. Después de colocar un stent con alta FA. CC: conducto cístico; CHC: conducto hepático común; T: tumor; VB: vesícula biliar. Modificado de: Nakai Y et al. J Gastroenterol Hepatol. 2014;29(7):1557-62. 
la aparición de colecistitis al evitar la oclusión del cístico por sobrecrecimiento tumoral (11).

El diámetro del stent puede afectar la incidencia de colecistitis. Los stents de menor diámetro tienen menos colecistitis. Se describe una tasa de colecistitis de 2,9 en stents de $8 \mathrm{~mm}$ frente al $7 \%$ en los de $10 \mathrm{~mm}$ y de $0 \%$ frente al $18 \%$ en los pacientes con obstrucción maligna del cístico (11). En nuestro estudio, todos los stents eran de $10 \mathrm{~mm}$ de diámetro.

Aunque el estudio sugiere que los stents cubiertos con baja FA se asocian con una baja incidencia de colecistitis y pancreatitis, la variable principal a evaluar es la permeabilidad de los stents. Se considera que un stent cubierto con baja FA se acomoda mejor a la curvatura del colédoco sin comprometer la permeabilidad del stent. El análisis de la permeabilidad del stent está por fuera del objetivo final de este estudio, pero el tiempo medio para reaparecer la obstrucción fue mayor para los stents con baja FA sobre los stents con alta FA ( $\mathrm{p}=0,048$; RR: 1,56; IC $95 \%: 1,01$ a 2,42 ) Por tanto, los stents con baja FA pueden disminuir las complicaciones como la colecistitis y pancreatitis manteniendo una mayor permeabilidad.

Un estudio mostró que la presencia de colelitiasis es un factor de riesgo para colecistitis (8), pero esto no se reprodujo en nuestro estudio. Esta diferencia podría estar explicada por la prevalencia tan alta en el estudio citado $(18,1$ $\%)$ y el estudio actual $(11,1 \%)$.

Se han intentado procedimientos empíricos con el fin de reducir la incidencia de la colecistitis, como el lavado transcístico de la vesícula biliar (8). Además, existen factores que predisponen a la sepsis como la desinfección inadecuada de los equipos, la inyección del contraste contaminado o el drenaje incompleto de la obstrucción, que pueden llevar a la colecistitis (22). En la práctica, un estudio previo documentó la infección por Pseudomonas después de la CPRE (23). Por tanto, deben administrarse antibióticos profilácticos a todos los pacientes así no exista un riesgo específico de infección (24). Un curso de antibióticos, a diferencia de una dosis profiláctica única, puede ser más eficaz para la prevención de la colecistitis si los pacientes han tenido factores de riesgo para esta.

La colecistostomía percutánea ha sido tradicionalmente el tratamiento de elección en pacientes con colecistitis aguda después del paso de un SBM por malignidad $(8,25-27)$. En el presente estudio se presentaron tres casos de colecistitis en el grupo con stent estándar, dos de ellas requirieron de la colecistostomía percutánea con apoyo radiológico y otra se resolvió espontáneamente. Otra alternativa de manejo, no implementada en este estudio, es el cambio de stent metálicos cubiertos por descubiertos o por stents plásticos (28).

La inserción percutánea del stent biliar se ha relacionado con menos posibilidad de desarrollar colecistitis, se atribuye esto a que no hay contaminación de la vía biliar, que es particularmente importante en los stents que cruzan la papila (7).
El presente estudio tiene algunas limitaciones. No se asignó al azar la colocación del stent, por lo que los sesgos de selección podrían estar presentes. El número de pacientes en ambos grupos es pequeño. El desenlace clínico con el uso de los stents puede variar con sus diferentes propiedades como el tipo de cobertura (silicona frente a poliuretano o politetrafluoroetileno), su estructura (trenzado o cortado por láser) o el tipo de alambre (acero o nitinol). Se requiere de un estudio prospectivo aleatorizado y controlado para confirmar la hipótesis de que los stents con baja FR pueden reducir la incidencia de colecistitis y/o pancreatitis.

\section{CONCLUSIONES}

El presente estudio confirma que el compromiso tumoral del cístico o del conducto de Wirsung es la principal causa en la génesis de la pancreatitis o la colecistitis y que esta se relaciona más frecuentemente con el uso de stents de alta FA, que incluso mostraron una menor permeabilidad a largo plazo. Es necesario un gran estudio prospectivo y aleatorizado para evaluar las implicaciones de los stents cubiertos en la génesis de la colecistitis y/o pancreatitis.

\section{Conflictos de intereses}

Ninguno de los autores presenta conflictos de intereses ni alianzas pecuniarias para declarar.

\section{Agradecimientos}

Expresamos nuestra gratitud a los Dr. J. Y. Song y Kang $S$ \& G Biotech Inc. de Seúl, Corea, por proveer de forma gratuita los stents de forma de flor para el desarrollo del presente estudio.

Este estudio se realizó con el apoyo del proyecto de sostenibilidad de la Vicerrectoría de Investigación de la Universidad de Antioquia.

\section{REFERENCIAS}

1. Castaño R, Álvarez O, García A, Quintero V, Sanín E, Erebrie $\mathrm{F}$, et al. Stent metálico versus plástico en la obstrucción biliar maligna distal. Rev Col Gastroenterol. 2009;24(3):248-55.

2. Sawas T, Al Halabi S, Parsi MA, Vargo JJ. Self-expandable metal stents versus plastic stents for malignant biliary obstruction: a meta-analysis. Gastrointest Endosc. 2015;82(2):256-267.e7. doi: 10.1016/j.gie.2015.03.1980.

3. Hong WD, Chen XW, Wu WZ, Zhu QH, Chen XR. Metal versus plastic stents for malignant biliary obstruction: an update meta-analysis. Clin Res Hepatol Gastroenterol. 2013;37(5):496-500. doi: 10.1016/j.clinre.2012.12.002.

4. Barkun AN, Adam V, Martel M, AlNaamani K, Moses PL. Partially covered self-expandable metal stents versus 
polyethylene stents for malignant biliary obstruction: a cost-effectiveness analysis. Can J Gastroenterol Hepatol. 2015;29(7):377-83.

5. Lee JH, Krishna SG, Singh A, Ladha HS, Slack RS, Ramireddy $S$, et al. Comparison of the utility of covered metal stents versus uncovered metal stents in the management of malignant biliary strictures in 749 patients. Gastrointest Endosc. 2013;78(2):312-24. doi: 10.1016/j.gie.2013.02.032.

6. Fumex F, Coumaros D, Napoleon B, Barthet M, Laugier R, Yzet T, et al. Similar performance but higher cholecystitis rate with covered biliary stents: results from a prospective multicenter evaluation. Endoscopy. 2006;38(8):787-92. doi: $10.1055 /$ s-2006-944515.

7. Isayama H, Kawabe T, Nakai Y, Tsujino T, Sasahira N, Yamamoto N, et al. Cholecystitis after metallic stent placement in patients with malignant distal biliary obstruction. Clin Gastroenterol Hepatol. 2006;4(9):1148-53. doi: 10.1016/j.cgh.2006.06.004.

8. Suk KT, Kim HS, Kim JW, Baik SK, Kwon SO, Kim HG, et al. Risk factors for cholecystitis after metal stent placement in malignant biliary obstruction. Gastrointest Endosc. 2006;64(4):522-9. doi: 10.1016/j.gie.2006.06.022.

9. Almadi MA, Barkun AN, Martel M. No benefit of covered vs uncovered self-expandable metal stents in patients with malignant distal biliary obstruction: a meta-analysis. Clin Gastroenterol Hepatol. 2013;11(1):27-37.e1. doi: 10.1016/j.cgh.2012.10.019.

10. Saleem A, Leggett CL, Murad MH, Baron TH. Meta-analysis of randomized trials comparing the patency of covered and uncovered self-expandable metal stents for palliation of distal malignant bile duct obstruction. Gastrointest Endosc. 2011;74(2):321-327.e1-3. doi: 10.1016/j.gie.2011.03.1249.

11. Nakai Y, Isayama H, Kawakubo K, Kogure H, Hamada T, Togawa $\mathrm{O}$, et al. Metallic stent with high axial force as a risk factor for cholecystitis in distal malignant biliary obstruction. J Gastroenterol Hepatol. 2014;29(7):1557-62. doi: 10.1111/jgh.12582.

12. Isayama H, Nakai Y, Toyokawa $\mathrm{Y}$, Togawa O, Gon C, Ito $Y$, et al. Measurement of radial and axial forces of biliary self-expandable metallic stents. Gastrointest Endosc. 2009;70(1):37-44. doi: 10.1016/j.gie.2008.09.032.

13. Isayama $H$, Nakai $Y$, Hamada T, Matsubara S, Kogure H, Koike K. Understanding the Mechanical forces of Self-Expandable Metal Stents in the Biliary Ducts. Curr Gastroenterol Rep. 2016;18(12):64. doi: 10.1007/s11894-016-0538-5.

14. Mukai T, Yasuda I, Isayama $H$, Nakashima M, Doi S, Iwashita Tet al. Comparison of axial force and cell width of self-expandable metallic stents: which type of stent is better suited for hilar biliary strictures? J Hepatobiliary Pancreat Sci. 2011;18(5):646-52. doi: 10.1007/s00534-011-0406-5.

15. Kawakubo K, Isayama $H$, Nakai Y, Togawa O, Sasahira N, Kogure $\mathrm{H}$, et al. Risk factors for pancreatitis following transpapillary self-expandable metal stent placement. Surg Endosc. 2012;26(3):771-6. doi: 10.1007/s00464-011-1950-4.

16. Shimizu S, Naitoh I, Nakazawa T, Hayashi K, Miyabe $\mathrm{K}$, Kondo $\mathrm{H}$, et al. Predictive factors for pancreatitis and cholecystitis in endoscopic covered metal stenting for distal malignant biliary obstruction. J Gastroenterol Hepatol. 2013;28(1):68-72. doi: 10.1111/j.14401746.2012.07283.x.

17. Nishida H, Inoue H, Ueno K, Nagata Y, Kato T, Miyazono $\mathrm{N}$, et al. Cholecystoduodenal fistula: a complication of inserted self-expandable metallic biliary stents. Cardiovasc Intervent Radiol. 1998;21(3):251-3.

18. Woo YS, Lee KH, Lee JK, Noh DH, Park JK, Lee KT, et al. Novel flower-type covered metal stent to prevent cholecystitis: experimental study in a pig model. Surg Endosc. 2016;30(3):1141-5. doi: 10.1007/s00464-015-4317-4.

19. Cotton PB, Eisen G, Romagnuolo J, Vargo J, Baron T, Tarnasky P, et al. Grading the complexity of endoscopic procedures: results of an ASGE working party. Gastrointest Endosc. 2011;73(5):868-74. doi: 10.1016/j. gie.2010.12.036.

20. Coté GA, Kumar N, Ansstas M, Edmundowicz SA, Jonnalagadda S, Mullady DK, et al. Risk of post-ERCP pancreatitis with placement of self-expandable metallic stents. Gastrointest Endosc. 2010;72(4):748-54. doi: 10.1016/j. gie.2010.05.023.

21. Nakai $Y$, Isayama $H$, Togawa $O$, Kogure $H$, Tsujino $T$, Yagioka $\mathrm{H}$, et al. New method of covered wallstents for distal malignant biliary obstruction to reduce early stent-related complications based on characteristics. Dig Endosc. 2011;23(1):49-55. doi: 10.1111/j.14431661.2010.01043.x.

22. Devière J, Motte S, Dumonceau JM, Serruys E, Thys JP, Cremer M. Septicemia after endoscopic retrograde cholangiopancreatography. Endoscopy. 1990;22(2):72-5.

23. Allen JI, Allen MO, Olson MM, Gerding DN, Shanholtzer CJ, Meier PB, et al. Pseudomonas infection of the biliary system resulting from use of a contaminated endoscope. Gastroenterology. 1987;92(3):759-63.

24. Bari K, Aslanian HR, Pollak J, Reiner E, Salem RR, Taddei $\mathrm{TH}$, et al. Emphysematous Cholecystitis Resulting in Secondary Biliary Cirrhosis: A Rare Complication of Endoscopic Retrograde Cholangiopancreatography. ACG Case Rep J. 2013;1(1):51-4. doi: 10.14309/crj.2013.18.

25. Ainley CC, Williams SJ, Smith AC, Hatfield AR, Russell $\mathrm{RC}$, Lees WR. Gallbladder sepsis after stent insertion for bile duct obstruction: management by percutaneous cholecystostomy. Br J Surg. 1991;78(8):961-3.

26. Vingan HL, Wohlgemuth SD, Bell JS 3rd. Percutaneous cholecystostomy drainage for the treatment of acute emphysematous cholecystitis. AJR Am J Roentgenol. 1990;155(5):1013-4. doi: 10.2214/ajr.155.5.2120927.

27. Dolan R, Pinkas H, Brady PG. Acute cholecystitis after palliative stenting for malignant obstruction of the biliary tree. Gastrointest Endosc. 1993;39(3):447-9.

28. Saxena P, Singh VK, Lennon AM, Okolo PI, Kalloo AN, Khashab MA. Endoscopic management of acute cholecystitis after metal stent placement in patients with malignant biliary obstruction: a case series. Gastrointest Endosc. 2013;78(1):175-8. doi: 10.1016/j.gie.2013.02.038. 